\title{
Importance of Cohort Study in Military Medicine: An International Overview
}

\author{
Milad Nazarzadeh ${ }^{1, *}$ \\ ${ }^{1}$ Iranian Research Center on Healthy Aging, Sabzevar University of Medical Sciences, Sabzevar, IR Iran \\ ${ }^{*}$ Corresponding author: Milad Nazarzadeh, Iranian Research Center on Healthy Aging, Sabzevar University of Medical Sciences, Sabzevar, IR Iran. Tel: +98-5144419572,
} Fax: +98-5144445648, E-mail: nazarzadeh_milad@yahoo.com

Received: April 23, 2015; Accepted: May 1, 2015

\section{Dear Editor,}

Observational studies have a great place in the modern research. Randomized controlled trials are not always the best choice or ethical solution to address some research questions in military medicine. Moreover, the validity of small size sampled cross-sectional and casecontrol studies, which usually lack quality control and use paper base questionnaires for main data collection, are under question. Mostly, the results of these small studies are not practical and confined to the shelves of libraries. On the other hand, a well-defined cohort study with combination of questionnaires and biologic sample for data collection, even with small sample size, can provide strong and valid results (1).

In a classical cohort study, first a disease-free population is identified by the status of exposure of interest and followed during time until the disease of interest happens. The military environment provides unique opportunity for cohort studies on military personnel and students. Because this population is always available, follow up is easy, and medical documents are collected in recruitment time. In the first stage, with standardization of routine data collection forms and in advanced stages with collecting biologic samples, the possibility of cohort studies is provided.

The importance of cohort studies in military population is growing worldwide. Our electronic search in PubMed database shows the growing trend of publications that mentioned 'military' and 'cohort' words in their title and abstract. Figure 1 shows the number of publications (across time) that mentioned the cohort study design and military related health issue in the world. The figure shows significant jump in the number of publication since 2002. Also, as the number of published papers may be biased criteria for scientometrics, we plotted the median of citation time of published papers during 50 years for military related publications with cohort design (worldwide) compared to the published articles from Swedish Twin Registry (one of the largest studies in the field of medical sciences)(Figure2).

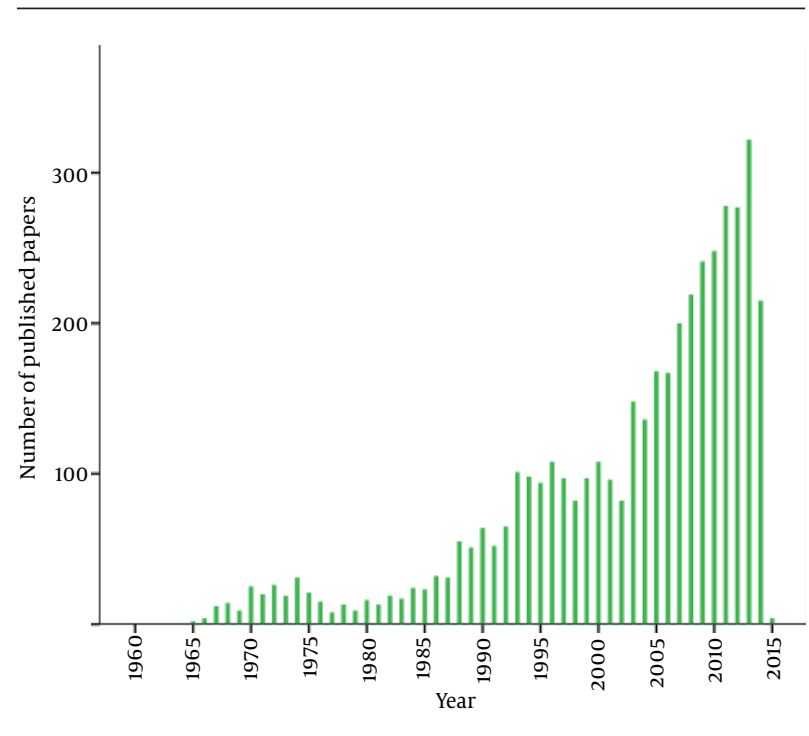

Figure 1. The Number of Publications by Year That Mentioned the Cohort Study Design and Military Related Health Issue Worldwide

Copyright (C) 2015, AJA University of Medical Sciences. This is an open-access article distributed under the terms of the Creative Commons Attribution-NonCommercial 4.0 International License (http://creativecommons.org/licenses/by-nc/4.0/) which permits copy and redistribute the material just in noncommercial usages, provided the original work is properly cited. 
Figure 2. The Median of Citation Time of Published Papers During 50 Years for Military Related Publication With Cohort Design (Worldwide) in Comparison With Swedish Twin Registry Published Papers

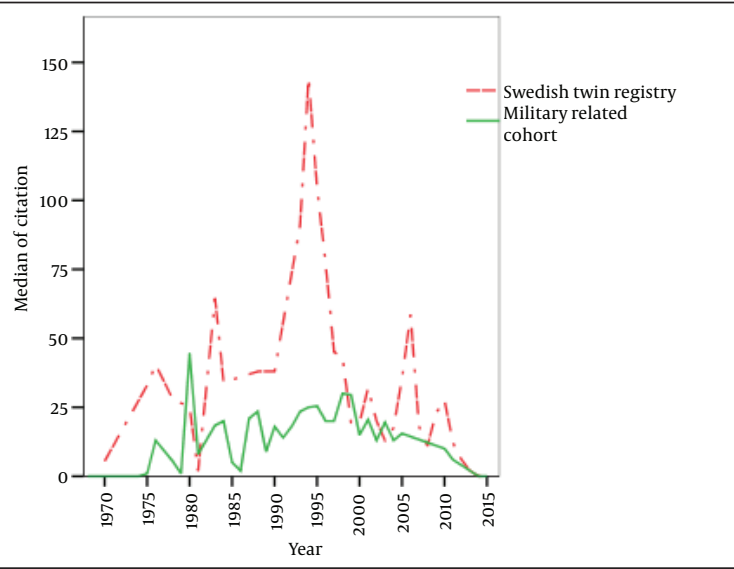

The result is based on Scopus database up to April 30, 2015 with the following search strategy: for military related publication with cohort design: (TITLE-ABS-KEY (\{Military\}) AND TITLE-ABS-KEY (\{cohort\}) OR TITLE-ABS-KEY (\{cohort study\})); and for Swedish Twin Registry: (TITLE-ABS-KEY ("Swedish Twin Register") OR TITLE-ABS-KEY (\{Swedish Twin Register\}) OR TITLE-ABSKEY (Swedish Twin Register) OR TITLE-ABS-KEY ("Swedish Twin”) OR TITLEABS-KEY (Swedish W/15 Twin W/15 Register) OR TITLE-ABS-KEY(Swedish PRE/3 Twin PRE/3 Register) OR TITLE-ABS-KEY (Swedish* Twin* Register* ) OR TITLEABS-KEY (Swedish W/15 Twin) OR TITLE-ABS-KEY(Swedish PRE/3 Twin).
In this figure, the importance of an epidemiologic design (cohort study) in military medicine field for production of science is clearly exhibited.

The result is based on PubMed database up to April 30, 2015 with the following search strategy: "Military Personnel” [Mesh] OR "Military Family" [Mesh] OR "Military Facilities" [Mesh] OR "Psychology, Military" [Mesh] OR "Military Science" [Mesh] OR "Military Psychiatry" [Mesh] OR "Military Nursing” [Mesh] OR “Military Medicine” [Mesh] OR “Military Hygiene” [Mesh] OR "Military Dentistry” [Mesh] OR “Hospitals, Military” [Mesh]) AND "Cohort Studies" [Mesh].

In conclusion, valid data collection with scientific research design is a must to respond to the medical needs of the military personnel. Along with the growth of military medical sciences in the world, the execution of well-conducted cohort studies on military personnel can be considered a research priority for Iranian medical military universities.

\section{References}

1. Harvey SB, Hatch SL, Jones M, Hull L, Jones N, Greenberg N, et al. The long-term consequences of military deployment: a 5-year cohort study of United kingdom reservists deployed to Iraq in 2003. Am J Epidemiol. 2012;176(12):1177-84. 